\title{
Risks and benefits in using zoledronic acid in patients with bone metastases
}

\author{
Dalia H. Zayed and Niveen A. AboTouk
}

\section{${ }^{1}$ Department of Clinical Oncology and Nuclear Medicine, Faculty of Medicine, Mansoura University, Egypt.}

Purpose: The aim of this study is to evaluate the efficacy and safety of zoledronic acid (4 mg over a 15-minute infusion) in patients with bone metastases.

Methods: Forty two patients with documented bone metastases from solid tumours and multiple myeloma were enrolled in this study during the period from September 2008 to October 2010. Zoledronic acid $4 \mathrm{mg}$ was administered intravenously in $100 \mathrm{ml}$ of $0.9 \%$ sodium chloride as 15 -minute infusion every 4 weeks.

Results: Prior to study entry, the majority of patients (76.2 \%) had an ECOG performance status score $\leq 1$. Twenty five patients (59.5\%) had experienced skeletal related events (SRE), 85.7\% of the patients suffered from pain and $81.0 \%$ used analgesics. During the period of treatment, 15 out of 42 patients $(35.7 \%)$ developed at least one SRE. The median time to first SRE was 25.22 months. Twenty six out of 36 patients who complained of pain at base line $(72.2 \%)$ had decreased in pain score. Six patients $(16.7 \%)$ had no changes while $4(11.1 \%)$ patients suffered from increasing in pain score. The most frequent side effect was hypocalcemia (28.5\%). Two patients had chills (4.8\%) and 4 patients had pyrexia $(9.5 \%)$ which occurred following the first injection. Serum creatinine level increased in 2 patients $(4.8 \%)$ and only one patient developed osteonecrosis of jaw.

Conclusion: Zoledronic acid reduces the incidence of skeletal related events (SREs), pain and hypercalcemia in patients with radiologic evidence of bone metastases. Accurate monitoring of ionized calcium and creatinine levels is mandatory and patients at -risk should also receive appropriate prophylactic attention to maintain oral health.

Key words: bone metastases - bisphosphonates - zoledronic acid -SREs.

Corresponding Author: Dalia Hatem Zayed.

E-mail: dhmzayed@gmail.com

\section{INTRODUCTION}

Bone metastases are a frequent complication of cancer, occurring in up to 70 percent of patients with advanced breast or prostate cancer ${ }^{1}$ and in approximately 15 to 30 percent of patients with carcinoma of the lung, colon, stomach, bladder, uterus, rectum, thyroid, or kidney. Furthermore, once tumors metastasize to bone, they are usually incurable: only 20 percent of patients with breast cancer are still alive five years after the discovery of bone metastasis ${ }^{2}$.

Bone metastases lead to a variety of complications, including severe bone pain, pathologic fractures, spinal cord compression, and hypercalcemia of malignancy (HCM), as well as the need for surgery or radiation therapy ${ }^{3,4}$. The associated morbidity can thus severely impact health-care resources and patient quality of life, particularly for those experiencing prolonged survival ${ }^{5}$.

Bisphosphonates (BP) are synthetic analogues of pyrophosphate, a natural regulator of bone metabolism found abundantly in bone matrix ${ }^{6}$.These compounds inhibit the differentiation of osteoclastic precursors, induce apoptosis of osteoclasts, and stimulate the release of osteoclastic inhibitory factor from osteoblasts ${ }^{7}$.

Zoledronic acid is a highly effective, nitrogencontaining bisphosphonate that has been approved for the treatment of HCM and for the prevention of skeletal complications secondary to multiple myeloma and bone metastases from any solid tumor ${ }^{8,9}$.

Zoledronic acid is generally well tolerated and induces just mild transient side effects, such as flu-like symptoms (fever, bone pain, arthralgia, nausea and vomiting), following the initial infusion. Over the last years, concerns of bisphosphonateinduced osteonecrosis of the jaw have emerged ${ }^{10}$. Furthermore, all bisphosphonates are known to be associated with laboratory abnormalities, particularly, elevated serum creatinine levels and hypocalcemia. Sporadic episodes of acute and subacute renal failure have been reported whereas hypocalcemia has not yet been the subject of detailed research $^{11}$. 
The aim of this study is to evaluate the efficacy and safety of zoledronic acid (4 mg over a 15-minute infusion) in patients with bone metastases.

\section{PATIENTS AND METHODS}

\section{Eligibility Criteria:}

Forty two patients with documented bone metastases from solid tumours and multiple myeloma who attended the department of Clinical Oncology \& Nuclear Medicine, Mansoura University Hospital from the period of September 2008 to October 2010were enrolled in this study.

Bone metastases were generally diagnosed by bone scintigraphy and $\mathrm{x}$ - rays, if spines were involved, magnetic resonance imaging (MRI) or computed tomography (CT) was used for further evaluation.

Patients were recruited if they had a baseline Eastern Cooperative Oncology Group (ECOG) performance status $\leq 2^{12}$, adequate liver function (determined by liver enzymes and serum bilirubin); and normal renal function (determined by serum creatinine $<1.5$ times the upper limit of normal and creatinine clearance $>60 \mathrm{ml} /$ minute).

\section{Treatment administration:}

Zoledronic acid (Zometa) $4 \mathrm{mg}$ was administered intravenously in $100 \mathrm{ml}$ of $0.9 \%$ sodium chloride as 15-minute infusion every 4 weeks. Supplementations with oral calcium $500 \mathrm{mg} /$ day and tablets containing 400-500 IU vitamin D /day were prescribed throughout treatment. Concomitant anticancer therapy according to the decision of the treatment panel was allowed, however the use of other bisphosphonates was prohibited.

\section{Assessments of patients:}

The efficacy of zoledronic acid was evaluated in this study, by analyzing skeletal related events (SREs), pain score, and analgesics use.

SREs were assessed at each visit and it was defined as: pathologic fractures, HCM, spinal cord compression, surgery or radiotherapy to bones. The distribution of each type of SRE, the proportion of patients with at least one SRE and the time to first SRE were analyzed.

Pain score for each patient was reported on a scale of 0 (none) to 3(severe pain interfering with activities of daily living) according to the National Cancer Institute (NCI) Common Toxicity Criteria Version $3.0^{13}$, and patients using analgesics were documented too.

Acute inflammatory reactions following the initial infusion including: bone pain, chills, pyrexia, nausea and vomiting were recorded.
Serum creatinine and calcium levels were requested before each administration of zoledronic acid. Abnormal calcium and creatinine serum levels were assessed according to the National Cancer Institute (NCI) Common Toxicity Criteria, Version $3.0^{13}$. Normal serum calcium is in the range of $8.5-10.5 \mathrm{mg} / \mathrm{dl}$. Hypocalcemia was defined as $\mathrm{G} 1$ when serum calcium levels were $<$ LLN-8.0 mg/dl. G2 when they were $<8.0-7.0 \mathrm{mg} / \mathrm{dl}$, G3 when they were $<7.0-6.0 \mathrm{mg} / \mathrm{dl}$, and G4 when they were $<6.0 \mathrm{mg} / \mathrm{dl}$.

The creatinine normal range is $0.60-1.20 \mathrm{mg} / \mathrm{dl}$. Increased serum creatinine was classified as follows based on the NCI Common Toxicity Criteria, Version 3.0: G1 when the creatinine level was $\leq 1.5 \times$ the upper limit of normal (ULN), G2 when the creatinine level was $>1.5-3.0 \times \mathrm{ULN}, \mathrm{G} 3$ when the creatinine level was $>3.0-6.0 \times \mathrm{ULN}$, and G4 when the creatinine level was $>6.0 \times$ ULN.

Patients were subjected to full dental evaluation. Follow up by radiography and clinical examination were done every 6 months or when there a complaint of toothache, orofacial pain or foul- smell oral secretions.

Zoledronic acid was temporarily stopped if patients had G2-G3 creatinine and calcium abnormalities and was resumed when levels returned to normal or within the G1range. Zoledronic acid was given until a substantial decline in the patient's performance status was observed ${ }^{5}$. Treatment was also stopped in cases of persistent G2 electrolyte abnormalities, gradual worsening of these parameters, or whenever any significant adverse event occurred.

\section{Statistical analysis:}

Statistical analysis was done using the SPSS (Statistical Package for Social Science) program version 15. The data included patients' age at the start of zoledronic acid treatment, gender, primary cancer types, ECOG performance status, previous occurrence of any bone events, specific anticancer therapy at the beginning of zoledronic acid treatment, the laboratory values at each dose administration, and side effects of treatment. The qualitative data were presented in the form of number and percentage. Quantitative data were presented as mean \pm standard deviation (SD). Tolerability, measured as an assessment of safety, was based on the frequency of side effects and abnormal laboratory values. Time to first SRE was analyzed by using Kaplan-Meier method.

\section{RESULTS}

The baseline characteristics of 42 patients enrolled in this study are shown in Table (1). The mean age at the start of treatment was 49. 9 years (range, $35-70$ 
years); the patients included 34 females $(81.0 \%)$ and 8 males $(19.0 \%)$. As regard primary cancer types, the patients comprised 31 breast cancer patients $(73.8 \%)$, 5 patients with multiple myeloma $(11.9 \%)$, and 3 prostate cancer patients $(7.1 \%)$. In addition, 2 patients had hepatoma (4.8\%), and only one patient had thyroid cancer.

Prior to study entry, the majority of patients $(76.2 \%)$ had an ECOG performance status score $\leq 1$. Twenty five patients $(59.5 \%)$ had experienced skeletal related events (SRE), $85.7 \%$ of the patients suffered from pain and $81.0 \%$ used analgesics. At study entry, 5 patients $(11.9 \%)$ were not receiving concomitant anticancer therapy. A total of 17 patients $(40.5 \%)$ were receiving hormonal therapy, 7 patients $(16.7 \%)$ were receiving chemotherapy and 13 patients $(31.0 \%)$ were under treatment with both hormonal therapy and chemotherapy (Table 1).

All patients had received zoledronic acid, $4 \mathrm{mg}$ intravenously, every 4 weeks with a median duration of therapy 19 months (range, 2- 36 months). During the period of treatment, 15 out of 42 patients $(35.7 \%)$ developed at least one SRE. The distribution of them is shown in Table (2). Nine patients (21.4\%) received palliative bone radiation. Pathological bone fracture was detected in 3 patients $(7.1 \%)$ and spinal cord compression occurred in one patient. Bone surgery was done to 2 patients; that included spinal decompression for the patient with spinal cord compression and internal fixation for a patient with a pathological fracture. However hypercalcemia of malignancy had not been noticed in our patients during treatment. The median time to first SRE was 25.22 months (95\% confidence interval; 20.82- 29.62) as shown in Figure (1).

All patients were evaluated at the end of treatment regarding pain. Twenty six out of 36 patients who complained of pain at base line $(72.2 \%)$ had decreased in pain score. Six patients (16.7\%) had no changes while 4 $(11.1 \%)$ patients suffered from increasing in pain score. Consequently, analgesics use decreased in 24 patients out of 34 patients using them at base line $(70.6 \%)$. However no change occurred in the analgesic use in $29.4 \%$ of patients. Only one patient started to use analgesics during the zoledronic administration and he was not using it before.

Regarding the side effects of treatment, it was found that all toxicities of grade 1 and 2 (Table 3). The most frequent event was hypocalcemia $(28.5 \%)$ which was corrected by delaying the injection one week; during this period, patients were under calcium and vitamin D therapy. Bone pain was observed in 4 patients $(9.5 \%)$. Two patients had chills (4.8\%) and 4 patients had pyrexia
(9.5\%) which occurred following the first injection. Three patients $(7.1 \%)$ complained of nausea during administration of the drug while vomiting was reported in one patient only.

Serum creatinine level increased in 2 patients $(4.8 \%)$, one of them had multiple myeloma and the other one was a metastatic cancer breast patient under a platinum-based chemotherapy.

Only one patient developed osteonecrosis of jaw in the form of swelling that appeared after 14 months of zoledronic acid administration and proved to be nonmalignant by histopathology, and so zoledronic acid was stopped. She had a minor debridement procedures and antibiotic therapy. Such patient had cancer breast with multiple metastases in bone, she was under treatment with aromatase inhibitor, and she developed multiple lung metastases and became in a poor general condition, and then died of progressive disease.

Table 1: Patients characteristics

\begin{tabular}{|c|c|}
\hline Characteristics & $\begin{array}{c}\text { Patients }(n=42) \\
\text { No. }(\%)\end{array}$ \\
\hline \multicolumn{2}{|l|}{ Age (years) } \\
\hline Range & $(35-70)$ \\
\hline Mean \pm SD & $49.9 \pm 9.6$ \\
\hline \multicolumn{2}{|l|}{ Gender } \\
\hline Female & $34(81.0)$ \\
\hline Male & $8(19.0)$ \\
\hline \multicolumn{2}{|c|}{ Primary cancer types } \\
\hline Breast & $31(73.8)$ \\
\hline Multiple myeloma & $5(11.9)$ \\
\hline Prostate & $3(7.1)$ \\
\hline Liver & $2(4.8)$ \\
\hline Thyroid & $1(2.4)$ \\
\hline \multicolumn{2}{|c|}{ ECOG performance status } \\
\hline $0-1$ & $32(76.2)$ \\
\hline 2 & $10(23.8)$ \\
\hline \multicolumn{2}{|l|}{ Previous SRE } \\
\hline Absent & $17(40.5)$ \\
\hline Present & $25(59.5)$ \\
\hline \multicolumn{2}{|c|}{ Anticancer treatment } \\
\hline Hormonal & $17(40.5)$ \\
\hline Chemotherapy & $7(16.7)$ \\
\hline Chemohormonal & $13(31.0)$ \\
\hline No treatment & $5(11.9)$ \\
\hline \multicolumn{2}{|c|}{ Pain score at base line } \\
\hline 0 & $6(14.3)$ \\
\hline 1 & $11(26.2)$ \\
\hline 2 & $16(38.1)$ \\
\hline 3 & $9(21.4)$ \\
\hline \multicolumn{2}{|l|}{ Analgesic use } \\
\hline No & $8(19.0)$ \\
\hline Yes & $34(81.0)$ \\
\hline
\end{tabular}


Table 2: Skeletal-related events (SREs).

\begin{tabular}{ll}
\hline SRE & $\begin{array}{c}\text { No (\%) } \\
(\mathbf{n = 4 2})\end{array}$ \\
\hline Bone radiation & $9(21.4)$ \\
\hline Pathologic bone fracture & $3(7.1)$ \\
\hline Spinal cord compression & $1(2.4)$ \\
\hline Bone surgery & $2(4.8)$ \\
\hline Hypercalcemia of malignancy & $0(0.0)$ \\
\hline
\end{tabular}

Table 3: Adverse events occurring during treatment.

\begin{tabular}{lc}
\hline Toxicity & $\begin{array}{l}\text { No (\%) } \\
(\mathbf{n}=\mathbf{4 2})\end{array}$ \\
\hline Bone pain & $4(9.5)$ \\
\hline Chills & $2(4.8)$ \\
\hline Pyrexia & $4(9.5)$ \\
\hline Nausea & $3(7.1)$ \\
\hline Vomiting & $1(2.4)$ \\
\hline Hypocalcemia & $12(28.5)$ \\
\hline Serum creatinine changes & $2(4.8)$ \\
\hline Osteonecrosis of jaw & $1(2.4)$ \\
\hline
\end{tabular}

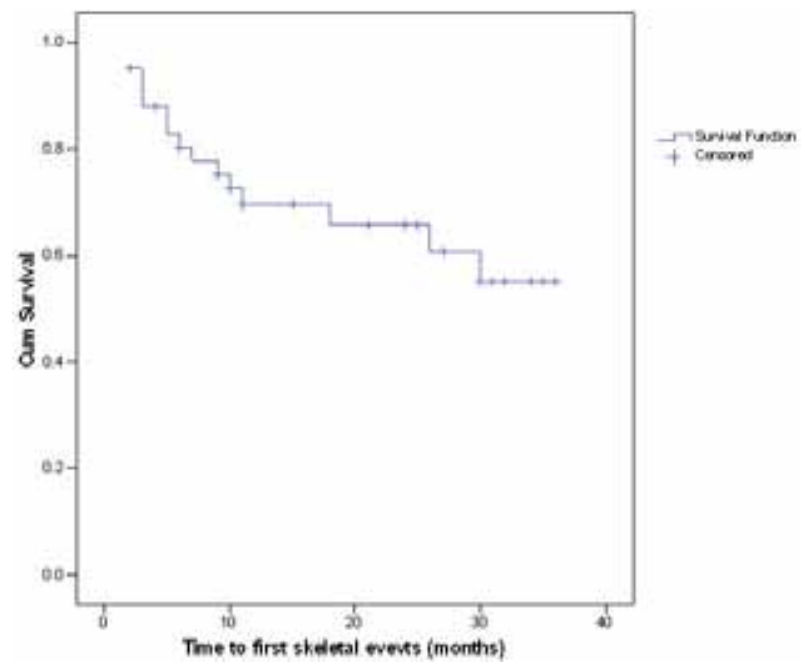

Figure 1: Time to first skeletal events.

\section{DISCUSSION}

Bisphosphonates (BPs) represent the current standard of care for the treatment of HCM and prevention of skeletal complications in patients with bone metastases. In particular, the American Society of Clinical Oncology
(ASCO) recommends pamidronate disodium and zoledronic acid for treating skeletal metastases in breast cancer and multiple myeloma (MM) patients (5). Furthermore, zoledronic acid is the first BP with proven efficacy in the treatment of bone metastases from solid tumors other than breast carcinoma ${ }^{14}$.

Of all the bisphosphonates available for the treatment of bone metastases, zoledronic acid is preferred because of the short infusion time ( 15 minutes). There is evidence from a large randomized trial on bone metastases from breast cancer and multiple myeloma that this drug ( $4 \mathrm{mg}$ i.v) is more effective than pamidronate in reducing the risk for skeletal complications both in the general population and in breast cancer patients ${ }^{15-18}$.

Because many cancer patients (particularly those with breast and prostate tumors) can experience prolonged survival durations, it may be useful to accumulate more data on the safety of BPs intravenously administered over a prolonged period, notably, in combination with anticancer agents. This unmet need is reinforced by ASCO guidelines that recommend the continuation of BP therapy until patient performance status deteriorates, even in the case of skeletal complications ${ }^{15}$ that is, skeletal-related events (SREs).

There is a scarcity of data on the incidence and types of SREs that occur in patients with metastatic bone disease with BPs for $>24$ months ${ }^{19}$. SREs were assessed in this study before and following a median period of zoledronic acid administration of 19 months. Twenty five patients $(59.5 \%)$ experienced SRE before zoledronic acid administration, and were thus at higher risk for subsequent SREs. Following zoledronic administration, the rate of SREs was reduced to $35.7 \%$ in which aligns with rates reported in the literature, ranging from $30 \%-49 \% 0^{8,20,21}$.

To assess the safety profile of the zoledronic acid, acute inflammatory reactions especially following the initial infusion, renal functions changes, hypocalcaemia and osteonecrosis of the jaw were recorded and analyzed in this study.

In our study, acute inflammatory reactions were observed in the form of fever $(9.5 \%)$, chills or rigors, bone pain $(9.5 \%)$, nausea $(7.1 \%)$ and vomiting. These results are much similar to that found by the retrospective study done on 312 patients, where fever was observed in $10 \%$ of patients and 5\% of them suffered from nausea and vomiting however, pyrexia occurred in $22 \%$ of patients which could be attributed to the underlying diseases ${ }^{22}$. Acute systemic inflammatory reaction is often self-limited and becomes less intense during subsequent treatments. Typically occurring within 48 hours of infusion, fever 
is usually low-grade, though occasionally associated with rigors. Accompanying bone pain may occur and usually starts about 12 hours after infusion, commonly felt in spines, ribs, and lower limbs, not necessarily near the site of metastases ${ }^{23,24}$. Supportive and symptomatic management with non steroidal anti-inflammatory drugs and acetaminophen is sufficient, although other serious causes of fever such as infection should first be excluded.

Hypocalcaemia was observed in $28.5 \%$ of patients receiving zoledronic acid and it was of grade 1 and 2 . The electrolyte imbalances especially hypocalcaemia may vary by the underlying disease. Among breast cancer patients treated in a phase III study of zoledronic acid, hypocalcaemia, though often mild, is observed in $30-35 \%$, compared to $7 \%$ in the placebo $\mathrm{arm}^{25}$.

We do not routinely assess the albumin level. It is therefore likely that some of the hypocalcemia occurrences which we registered were dependent on hypoalbuminemia, as proven by patients ionized calcium levels, but not all of them. It is therefore important that the albumin level is assessed in patients presenting with hypocalcemia during zoledronic acid therapy before making any decision regarding treatment. Alternatively, assessing ionized calcium may be useful in decision making.

Clearly, hypocalcemia can be minimized by strictly adhering to the recommended dosage and interval as high-dose or too short treatment interval increases the risk $^{26}$. Daily calcium and vitamin D supplementation is recommended during treatment with zoledronic acid, but hypocalcemia can still occur ${ }^{27}$.

The rate of changes in serum creatinine level was $4.5 \%$ of the patients in this study, and it was of grade $1-$ 2 only. These results are much similar to that found by large, randomized trials in patients with bone metastases from breast cancer, multiple myeloma, and prostate cancer, and other tumours. A clinically insignificant rise in serum creatinine level was observed in $<5 \%$ of patients, and grade 3-4 renal toxicities were extremely rare $^{28-30}$.

On the other hand, our results are less than that observed by three large studies done to evaluate the efficacy and safety of zoledronic acid with an incidence of increased serum creatinine ranging from 9\%$10 \%{ }^{16,17,31}$. This difference could be attributed to the small population sample in the current study. Moreover, the increased occurrence of renal impairment could be attributed to progression of the underlying disease as these three studies were done only on advanced multiple myeloma and breast cancer patients.
The risk of renal failure is directly related to the drug infusion time and dosage ${ }^{27}$. High-dose zoledronic acid with short infusion time is strongly nephrotoxic ${ }^{16,32,33}$. Using the recommended infusion time and dosage, decreases the rate of renal impairment. Previous treatments with bisphosphonates, advanced age and multiple cycles of therapies increases the risk, although safe treatment beyond 5years has been reported. Patients with baseline renal impairment also at increased the $\operatorname{risk}^{34,35}$

Therefore, prevention is crucial. This includes adequate hydration, checking serum creatinine before each infusion, avoiding concurrent nephrotoxic agents, reducing the dosage for patients with mild renal insufficiency (for zoledronic acid), and withholding treatment in the presence of renal deterioration ${ }^{35}$.

Only one patient (out of 42 patients) developed osteonecrosis of the lower jaw after 14 months of zoledronic acid administration in our study. This patient started to complain from orofacial pain in the form of toothache and trismus then a foul-smelling discharge from a cutaneous sinus appeared. The diagnoses was confirmed radiologically (demonstrating mottled bone) and histopathologically. Discontinuation of bisphosphonate was recommended. Appropriate antibiotics for osteomyelitis, chlorhexidine mouthwash, minor debridement, and wound irrigation have limited the progression of the lesion. Lastly, this patient died of progression of the metastatic disease. This side effect seems to be the most serious adverse event associated with this type of treatment, and the risk for its development increases with the duration of bisphosphonate use. It was also observed in patients treated with RT for head and neck tumors; other risk factors for osteonecrosis are long-term steroid therapy, cytotoxic chemotherapy, dental or sinus infections, and trauma ${ }^{36}$. The incidence is, at present, uncertain in the literature because most cases are self-reported or are found on retrospective analysis and it varies in the range of $0.03 \%-11 \%{ }^{37-39}$.

\section{CONCLUSION}

Zoledronic acid reduces the incidence of skeletal related events (SREs), pain and hypercalcemia in patients with radiologic evidence of bone metastases. This reinforces the ASCO recommendations to continue BP therapy even after the onset of an SRE. There is low incidence of renal functions changes, hypocalcemia and osteonecrosis, so accurate monitoring of ionized calcium and creatinine levels is mandatory and patients at -risk should also receive appropriate prophylactic attention to maintain oral health. Risks and benefits must be weighted carefully by both physicians and well-informed patients. 


\section{REFERRENCES}

1. Jemal A, Tiwari RC, Murray T, Ghafoor A, Samuels A, Ward E, et al. Cancer statistics, 2004. CA Cancer J.Clin. 2004;54(1):8-29.

2. Coleman RE. Metastatic bone disease: Clinical features, pathophysiology and treatment strategies. Cancer Treat. Rev. 2001;27(3):165-76.

3. Mercadante S. Malignant bone pain: Pathophysiology and treatment. Pain 1997;69(1-2):1-18.

4. Hillner BE, Ingle JN, Chlebowski RT, Gralow J, Yee GC, Janjan NA, et al. American Society of Clinical Oncology 2003 update on the role of bisphosphonates and bone health issues in women with breast cancer. J.Clin.Oncol. 2003;21(21):4042-57.

5. Weinfurt KP, Castel LD, Li Y, Timbie JW, Glendenning GA, Schulman KA. Health-related quality of life among patients with breast cancer receiving zoledronic acid or pamidronate disodium for metastatic bone lesions. Med. Care 2004;42(2):164-75.

6. Russell RG, Bisaz S, Fleisch H, Currey HL, Rubinstein HM, Dietz AA, et al. Inorganic pyrophosphate in plasma, urine, and synovial fluid of patients with pyrophosphate arthropathy (chondrocalcinosis or pseudogout). Lancet 1970;2(7679):899-902.

7. Rogers MJ, Gordon S, Benford HL, Coxon FP, Luckman $\mathrm{SP}$, Monkkonen J, et al. Cellular and molecular mechanisms of action of bisphosphonates. Cancer 2000;88(12 Suppl.):2961-78.

8. Coleman RE. Bisphosphonates: Clinical experience. Oncologist 2004;9(Suppl. 4):14-27.

9. Lipton A. Treatment of skeletal complications in cancer patients: Focus on bisphosphonates. Oncology Spec.Ed. 2004;7:59-64.

10. Migliorati CA, Schubert MM, Peterson DE, Seneda LM. Bisphosphonate-associated osteonecrosis of mandibular and maxillary bone: An emerging oral complication of supportive cancer therapy. Cancer 2005;104(1):83-93.

11. Zuradelli M, Masci G, Biancofiore G, Gullo G, Scorsetti M, Navarria $\mathrm{P}$, et al. High incidence of hypocalcemia and serum creatinine increase in patients with bone metastases treated with zoledronic acid. Oncologist 2009;14(5):548-56.

12. Oken MM, Creech RH, Davis TE. Toxicology and response criteria of the Eastern Cooperative Oncology Group. Am.J.Clin.Oncol.Cancer Clin.Trials 1982; 5(6):649-55.

13. Cancer Therapy Evaluation Program. Common terminology criteria for adverse events. 2006. Aug 9; Version 3.0, DCTD, NCI, NIH, DHHS.

14. Lacerna L, Hohneker J. Zoledronic acid for the treatment of bone metastases in patients with breast cancer and other solid tumors. Semin.Oncol. 2003;30(5 Suppl. 16):150-60.

15. Conte P, Guarneri V. Safety of intravenous and oral bisphosphonates and compliance with dosing regimens. Oncologist 2004;9(Suppl. 4):28-37.
16. Rosen LS, Gordon D, Tchekmedyian S, Yanagihara R, Hirsh V, Krzakowski M, et al. Zoledronic acid versus placebo in the treatment of skeletal metastases in patients with lung cancer and other solid tumors: A phase III, double-blind, randomized trial - The zoledronic acid lung cancer and other solid tumors study group. J.Clin.Oncol. 2003;21(16):3150-7.

17. Saad F, Gleason DM, Murray R, Tchekmedyian S, Venner $\mathrm{P}$, Lacombe $\mathrm{L}$, et al. A randomized, placebocontrolled trial of zoledronic acid in patients with hormone-refractory metastatic prostate carcinoma. J.Natl. Cancer Inst. 2002;94(19):1458-68.

18. Rosen L, Gordon D, Tchekmedyian S. Zoledronic acid (significantly reduces Skeletal-Related Events (SREs) in patients with bone metastases from solid tumors [abstract]. Proc.Am.Soc.Clin.Oncol. 2002;21:295a.

19. Hortobagyi GN. Progress in the management of bone metastases: One continent at a time? J.Clin.Oncol. 2005;23(15):3299-301.

20. Berenson JR, Rosen LS, Howell A, Porter L, Coleman RE, Morley W, et al. Zoledronic acid reduces skeletalrelated events in patients with osteolytic metastases: A double-blind, randomized dose-response study. Cancer 2001;91(7):1191-200.

21. Rosen LS, Gordon DH, Dugan Jr. W, Major P, Eisenberg PD, Provencher L, et al. Zoledronic acid is superior to pamidronate for the treatment of bone metastases in breast carcinoma patients with at least one osteolytic lesion. Cancer 2004;100(1):36-43.

22. Cartenì G, Bordonaro R, Giotta F, Lorusso V, Scalone S, Vinaccia $\mathrm{V}$, et al. Efficacy and safety of zoledronic acid in patients with breast cancer metastatic to bone: A multicenter clinical trial. Oncologist 2006;11(7):841-8.

23. Haworth CS, Selby PL, Webb AK, Mawer EB, Adams JE, Freemont TJ. Severe bone pain after intravenous pamidronate in adult patients with cystic fibrosis. Lancet 1998;352(9142):1753-4.

24. Adami S, Bhalla AK, Dorizzi R, Montesanti F, Rosini S, SalvagnoG, etal. The acute-phaseresponseafterbisphosphonate administration. Calcif.Tissue Int. 1987;41(6):326-31.

25. Kohno N, Aogi K, Minami H, Nakamura S, Asaga T, Iino $\mathrm{Y}$, et al. Zoledronic acid significantly reduces skeletal complications compared with placebo in Japanese women with bone metastases from breast cancer: A randomized, placebo-controlled trial. J.Clin.Oncol. 2005; 23(15):3314-21.

26. Breay S, Fergus C, Palmieri C, Dhillon T, Coombes C, Vigushin $\mathrm{D}$, et al. Hypocalcaemia after intravenous bisphosphonate. Br.Med.J. 2004;328(7453):1439-40.

27. Rosen LS, Gordon D, Kaminski M, Howell A, Belch A, Mackey J, et al. Long-term efficacy and safety of zoledronic acid compared with pamidronate disodium in the treatment of skeletal complications in patients with advanced multiple myeloma or breast carcinoma: A randomized, double-blind, multicenter, comparative trial. Cancer 2003;98(8):1735-44. 
28. Guarneri V, Donati S, Nicolini M, Conte P. Renal safety of zoledronic acid in patients with bone metastases from breast cancer or other tumors treated with intravenous bisphosphonates for up to ten years. Ann.Oncol. 2004;15(Suppl. 3):811p.

29. Rosen LS, Gordon D, Tchekmedyian NS, Yanagihara R, Hirsh V, Krzakowski M, et al. Long-term efficacy and safety of zoledronic acid in the treatment of skeletal metastases in patients with nonsmall cell lung carcinoma and other solid tumors: A randomized, phase III, double-blind, placebo-controlled trial. Cancer 2004;100(12):2613-21.

30. Lipton L. Bisphosphonate therapy for patients with breast cancer. Curr.Cancer Ther.Rev. 2005;1:217-25.

31. Rosen LS, Gordon D, Kaminski M, Howell A, Belch A, Mackey J, et al. Zoledronic acid versus pamidronate in the treatment of skeletal metastases in patients with breast cancer or osteolytic lesions of multiple myeloma: A phase III, double-blind, comparative trial. Cancer J. 2001;7(5):377-87.

32. Markowitz GS, Fine PL, Stack JI, Kunis CL, Radhakrishnan J, Palecki W, et al. Toxic acute tubular necrosis following treatment with zoledronate (Zometa). Kidney Int. 2003;64(1):281-9.
33. Pavlakis N, SchmidtR, Stockler M. Bisphosphonates for breast cancer. Cochrane Database Syst.Rev. 2002(3):CD003474.

34. Mazj S, Lichtman SM. Renal dysfunction associated with bisphosphonate use: retrospective analysis of 293 patients with respect to age and other clinical characteristics. Proc. Am.Soc.Clin.Oncol. 2004;23:735a.

35. Stein SH, Davidson R, Tweed A. Renal dysfunction with iv bisphosphonates in patients with metastatic breast cancer. Proc.Am.Soc.Clin.Oncol. 2003;22:745a.

36. Maerevoet MM, Lenoir JJ, Degols L. Ten cases of osteonecrosis of the jaws following a treatment by bisphosphonates within the framework of bone metastasis of breast cancer and multiple myeloma: Experience in summer 2004 of a single center. J Clin Oncol 2005:8271.

37. Vannucchi AM, Ficarra G, Antonioli E, Bosi A. Osteonecrosis of the jaw associated with zoledronate therapy in a patient with multiple myeloma. Br.J.Haematol. 2005;128(6):738.

38. Durie BGM, Katz M, Crowley J, Woo SB, Hande K, Richardson $\mathrm{PG}$, et al. Osteonecrosis of the jaw and bisphosphonates. N.Engl.J.Med. 2005;353(1):99-102.

39. Van den Wyngaert T, Huizing MT, Vermorken JB. Bisphosphonates and osteonecrosis of the jaw: Cause and effect or a post hoc fallacy? Ann.Oncol. 2006;17(8):1197-204. 